\title{
ON THE SUPPLEMENT TO THE LAW OF BIQUADRATIC RECIPROCITY
}

\author{
KENNETH S. WILLIAMS
}

ABstract. A short proof is given of the supplement to the law of biquadratic reciprocity proved by Eisenstein in 1844 .

If $\pi$ is a Gaussian prime, which is not an associate of $1+i$, then $N(\pi) \equiv 1(\bmod 4)$ and the biquadratic residue character of the Gaussian integer $\alpha$ modulo $\pi$ is defined by

(1) $\left(\frac{\alpha}{\pi}\right)_{4}= \begin{cases}0, & \text { if } \alpha \equiv 0(\bmod \pi), \\ i^{r}, & \text { if } \alpha \neq \equiv(\bmod \pi) \text { and } \alpha^{(N(\pi)-1) / 4} \equiv i^{r}(\bmod \pi), \\ & \text { with } r=0,1,2,3\end{cases}$

As Gaussian integers can be factored uniquely into primes, the Jacobi extension of this symbol is obtained by defining for any Gaussian integer $\tau \not \equiv 0(\bmod 1+i)$

(2) $\left(\frac{\alpha}{\tau}\right)_{4}= \begin{cases}1, & \text { if } \tau \text { is a unit, } \\ \left(\frac{\alpha}{\pi_{1}}\right)_{4} \cdots\left(\frac{\alpha}{\pi_{r}}\right)_{4}, & \text { if } \tau \text { is not a unit and } \tau=\pi_{1} \cdots \pi_{r} \\ & \text { where the } \pi_{i} \text { are primes. }\end{cases}$

If $\alpha, \beta, \tau, \rho$ are Gaussian integers with $\tau, \rho \not \equiv 0(\bmod 1+i)$ then it is easily verified that

$$
\left(\frac{\alpha}{\tau}\right)_{4}^{4}=\left\{\begin{array}{l}
1, \text { if }(\alpha, \tau)=1, \\
0, \text { if }(\alpha, \tau) \neq 1,
\end{array}\right\}, \overline{\left(\frac{\bar{\alpha}}{\tau}\right)_{4}}=\left(\frac{\alpha}{\tau}\right)_{4}^{3}=\left(\frac{\bar{\alpha}}{\bar{\tau}}\right)_{4}
$$

Received by the editors January 31, 1975.

AMS (MOS) subject classifications (1970). Primary 10A15.

Key words and phrases. Gaussian integers, biquadratic residues, primary integers, biquadratic reciprocity. 


$$
\left(\frac{\alpha \beta}{\tau}\right)_{4}=\left(\frac{\alpha}{\tau}\right)_{4}\left(\frac{\beta}{\tau}\right)_{4}, \quad\left(\frac{\alpha}{\tau \rho}\right)_{4}=\left(\frac{\alpha}{\tau}\right)_{4}\left(\frac{\alpha}{\rho}\right)_{4},
$$

and

$$
(\alpha / \tau)_{4}=(\beta / \tau)_{4} \quad \text { if } \alpha \equiv \beta \quad(\bmod \tau)
$$

Also we have

$$
(i / \tau)_{4}=i^{(N(\tau)-1) / 4}
$$

so that in particular if $k$ is a rational integer $\equiv 1(\bmod 4)$ then

$$
(i / k)_{4}=(-1)^{(k-1) / 4} \text {. }
$$

It is also easy to show that if $a$ and $k$ are rational integers with $(a, k)=1, k$ odd, then

$$
(a / k)_{4}=+1
$$

(See [5, p. 143] for (7) and (8).)

A Gaussian integer $a+b i$ will be called primary if

$$
a+b i \equiv 1 \quad\left(\bmod (1+i)^{3}\right)
$$

equivalently $a+b-1 \equiv 0(\bmod 4)$ and $b \equiv 0(\bmod 2)$. A product of primary Gaussian integers is clearly also primary. If a Gaussian integer is not divisible by $1+i$, then among its four associates exactly one is primary. No multiple of $1+i$ can of course be primary. If $a+b i$ is primary it is convenient to set $a^{*}=(-1)^{b / 2} a$ so that

$$
a^{*} \equiv 1 \quad(\bmod 4), \quad \frac{a^{*}-1}{2} \equiv \frac{a-1}{2}+\frac{b^{2}}{4} \quad(\bmod 4) .
$$

Also from (6) with $a+b i$ primary we obtain

$$
(i /(a+b i))_{4}=i^{-(a-1) / 2} .
$$

We are now in a position to state (see, for example, [3, p. 106])

THE LAW OF BIQUADRATIC RECIPROCITY. If $\alpha=a+b i, \beta=c+d i$ are primary Gaussian integers, then

$$
(\alpha / \beta)_{4}=(-1)^{b d / 4}(\beta / \alpha)_{4}
$$

This law was first formulated by Gauss [2] and later proved by Jacobi [4] and Eisenstein [1]. More recently a proof of it has been given by Kaplan [5]. 
The purpose of this note is to give a simple presentation of the complementary theorem to the law of biquadratic reciprocity relating to the prime $1+i$. The proof uses a special case of (11) namely: if $k$ is a rational integer $\equiv 1(\bmod 4)$ and $\gamma$ is a primary Gaussian integer then

$$
(k / \gamma)_{4}=(\gamma / k)_{4}
$$

SUPPLEMENT TO THE LAW OF BIQUADRATIC RECIPROCITY. If $\alpha=c+d i$ is a primary Gaussian integer then

$$
((1+i) / \alpha)_{4}=i^{\left((c+d)-(1+d)^{2}\right) / 4} .
$$

(For this formulation see, for example, [6, p. 77].)

Proof. We first establish that if $k$ is a rational integer $\equiv 1(\bmod 4)$ then

$$
((1+i) / k)_{4}=i^{(k-1) / 4} \text {. }
$$

If $k_{1}, k_{2}$ are rational integers $\equiv 1(\bmod 4)$ then

$$
\frac{k_{1}-1}{4}+\frac{k_{2}-1}{4} \equiv \frac{k_{1} k_{2}-1}{4}(\bmod 4)
$$

so that by (4), as (13) is trivially true when $k=1$, it suffices to prove (13) for (i) $k=p($ prime $) \equiv 1(\bmod 4)$, and (ii) $k=-q, q($ prime $) \equiv 3(\bmod 4)$.

(i) We have $p=\pi \bar{\pi}$, where $\pi, \bar{\pi}$ are primary Gaussian primes, so that

$$
\begin{aligned}
\left(\frac{1+i}{p}\right)_{4} & =\left(\frac{1+i}{\pi}\right)_{4}\left(\frac{1+i}{\bar{\pi}}\right)_{4}=\left(\frac{1+i}{\pi}\right)_{4}\left(\frac{i}{\bar{\pi}}\right)_{4}\left(\frac{1-i}{\bar{\pi}}\right)_{4} \\
& =\left(\frac{i}{\bar{\pi}}\right)_{4}\left(\frac{1+i}{\pi}\right)_{4} \overline{\left(\frac{1+i}{\pi}\right)_{4}}=\left(\frac{i}{\bar{\pi}}\right)_{4}=i^{(p-1) / 4}
\end{aligned}
$$

(ii) Working modulo $q$ we have

$$
\begin{aligned}
\left(\frac{1+i}{-q}\right)_{4} & \equiv(1+i)^{\left(q^{2}-1\right) / 4} \equiv(2 i)^{\left(q^{2}-1\right) / 8} \equiv\left(2^{(q-1) / 2}\right)^{(q+1) / 4} i^{\left(q^{2}-1\right) / 8} \\
& \equiv\left((-1)^{(q+1) / 4}\right)^{(q+1) / 4} i^{\left(q^{2}-1\right) / 8} \equiv(-1)^{(q+1) / 4} i^{\left(q^{2}-1\right) / 8} \\
& \equiv i^{(q+1) / 2+\left(q^{2}-1\right) / 8} \equiv i^{(-q-1) / 4}
\end{aligned}
$$

so that

$$
((1+i) /-q)_{4}=i^{(-q-1) / 4} \text {. }
$$

This completes the proof of (13).

Now set $\alpha=c+d i=k(a+b i)$, where $(a, b)=1$ and $k \equiv 1(\bmod 4)$, so that $a+b i$ is primary. Then we have 


$$
\begin{aligned}
\left(\frac{1+i}{a+b i}\right)_{4} & =\left(\frac{i}{a^{*}}\right)_{4}^{3}\left(\frac{b i}{a^{*}}\right)_{4}\left(\frac{1+i}{a+b i}\right)_{4} \\
& =\left\{(-1)^{\left(a^{*}-1\right) / 4}\right\}^{3}\left(\frac{a+b i}{a^{*}}\right)_{4}\left(\frac{1+i}{a+b i}\right)_{4} \\
& =(-1)^{\left(a^{*}-1\right) / 4}\left(\frac{a^{*}}{a+b i}\right)_{4}\left(\frac{1+i}{a+b i}\right)_{4} \\
& =i^{\left(a^{*}-1\right) / 2}\left(\frac{i}{a+b i}\right)_{4}^{b}\left(\frac{a+a i}{a+b i}\right)_{4} \\
& =i^{(a-1) / 2+b^{2} / 4+b^{2} / 2}\left(\frac{i(a-b)}{a+b i}\right)_{4} \\
& =i^{3 b^{2} / 4}\left(\frac{a-b}{a+b i}\right)_{4} \\
& =i^{-b^{2} / 4}\left(\frac{a+b i}{a-b}\right)_{4} \\
& =i^{-b^{2} / 4}\left(\frac{b}{a-b}\right)_{4}\left(\frac{1+i}{a-b}\right)_{4} \\
& =i^{-b^{2} / 4+(a-b-1) / 4} \\
& =i^{\left((a+b)-(1+b)^{2}\right) / 4}
\end{aligned}
$$

so that

$$
\begin{aligned}
\left(\frac{1+i}{\alpha}\right)_{4} & =\left(\frac{1+i}{k}\right)_{4}\left(\frac{1+i}{a+b i}\right)_{4} \\
& =i^{(k-1) / 4+\left(a+b-(1+b)^{2}\right) / 4} \\
& =i^{\left(k a+k b-(1+k b)^{2}\right) / 4} \\
& =i^{\left(c+d-(1+d)^{2}\right) / 4}
\end{aligned}
$$

\section{REFERENCES}

1. G. Eisenstein, (i) Lois de reciprocité, J. Reine Angew. Math. 28 (1844), 53-67.

(ii) Einfacher Beweis und Verallgemeinerung des Fundamentaltheorems für die biquadratischen Reste, J. Reine Angew. Math. 28 (1844), 223-245.

2. C. F. Gauss, (i) Theoria residuorum biquadraticorum. I, Göttinger Abh. 6 (1828);

(ii) Theoria residuorum biquadraticorum. II, Göttinger Abh. 7 (1832).

3. H. Hasse, Bericht über neuere Untersuchungen und Probleme aus der Theorie der algebraischen Zahlkörper. Teil II: Reziprozitätsgesetz, 2nd. rev. ed., Physica-Verlag, Würzburg-Vienna, 1965. MR 33 \# 4045b.

4. C. G. J. Jacobi, Uber die Kreisteilung und ihre Anwendung auf die Zahlentheorie, J. Reine Angew. Math. 30 (1846), 166-182.

5. Pierre Kaplan, Démonstration des lois de réciprocité quadratique et biquadratique, J. Fac. Sci. Tokyo Sect. I 16 (1969), 115-145. MR 41 \#1683.

6. H. J. S. Smith, Report on the theory of numbers, Chelsea, New York, 1965.

Department of Mathematics, Carleton University, Ottawa, Ontario, Canada 